\title{
Real-time and Freehand Multimodal Imaging: Combining White Light Endoscopy with All-Optical Ultrasound
}

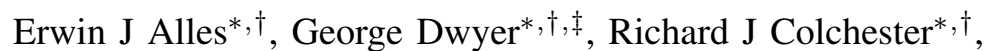 \\ Efthymios Maneas ${ }^{*, \dagger}$, Danail Stoyanov ${ }^{\dagger, \ddagger, \S}$ and Adrien E Desjardins, ${ }^{*} \dagger$ \\ * Department of Medical Physics \& Biomedical Engineering, University College London, London, UK \\ ${ }^{\dagger}$ Wellcome / EPSRC Centre for Interventional and Surgical Sciences, University College London, London, UK \\ $\ddagger$ UCL Robotics Institute, University College London, London, UK

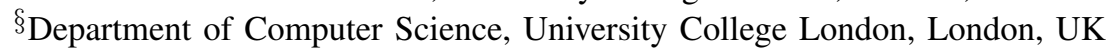 \\ E-mail: e.alles@ucl.ac.uk
}

\begin{abstract}
Minimally invasive surgery offers significant benefits over open surgery in terms of patient recovery, complication rates, and cost. Accurate visualisation is key for successful interventions; however, no single imaging modality offers sufficient resolution, penetration, and soft-tissue contrast to adequately monitor interventional treatment. Consequently, multimodal interventional imaging is intensively investigated. All-optical ultrasound (AOUS) imaging is an emerging modality where light is used to both generate and detect ultrasound. Using fibre-optics, highly miniaturised imaging probes can be fabricated that yield high-quality pulse-echo images and are readily integrated into minimally invasive interventional instruments. In this work, we present the integration of a miniature (diameter: $800 \mu \mathrm{m}$ ), highly directional AOUS imaging probe into a commercially available white light urethroscope, and demonstrate the first real-time, 3D multimodal imaging combining AOUS and white light endoscopy. Through the addition of an electromagnetic tracker, the position and pose of the instrument could be continuously recorded. This facilitated accurate real-time registration of the imaging modalities, as well as freehand operation of the instrument. In addition, the freehand imaging paradigm allowed for "piece-wise" scanning where the instrument was retracted and repositioned without recalibration. The presented imaging probe and system could significantly improve the quality of image guidance during interventional surgery.

Index Terms-Multimodal imaging, all-optical ultrasound imaging, endoscopy, freehand 3D imaging
\end{abstract}

\section{INTRODUCTION}

Minimally invasive (or interventional) surgery offers significant benefits over open surgery: patients benefit from less scarring, quicker recovery, lower complication rates, and reduced procedural costs [1]. Accurate visualisation is key for successful interventions; however, no single imaging modality offers sufficient resolution, penetration, and soft-tissue contrast to adequately monitor interventional treatment [2]. Practical

This work was supported by the Wellcome/EPSRC Centre for Interventional and Surgical Sciences (WEISS) (203145Z/16/Z), the Engineering and Physical Sciences Research Council (EPSRC) (EP/N021177/1, EP/L016478/1, EP/N027078/1, EP/R004080/1, EP/P012841/1, NS/A000027/1), the Wellcome Trust (WT101957), and the Royal Academy of Engineering via the Chair in Emerging Technologies (CiET1819/2/36) and the Research Fellowship schemes. considerations pose additional challenges: blood or debris generated during surgery occlude the view during white light endoscopy, ionising radiation prevents longitudinal use of $\mathrm{X}$ ray imaging, ultrasound lacks tissue specificity, and interventional instruments are typically incompatible with magnetic resonance imaging (MRI).

Multimodal imaging, where the properties and advantages of multiple modalities are combined to overcome modalityspecific limitations, is the subject of intensive study. In an interventional setting, where imaging depths of several centimetres are required, this is typically performed by combining an "external" imaging modality (e.g., MRI, X-ray) with an endoscopic imaging modality (e.g., ultrasound or white light endoscopy). However, accurate and efficient registration of such images in real-time remains challenging [3]. In addition, combining external and endoscopic imaging modalities can cause electromagnetic or mechanical interference and incompatibility, and requires exclusive use of imaging facilities that are usually in high demand. Instead, in this work we focus on multimodal imaging achieved using a single interventional instrument. This approach offers automatic co-registration, a reduced technological and experimental complexity, a simplified clinical workflow, and requires less clinical staff (and hence reduced costs) to operate equipment.

In this work, an all-optical ultrasound (AOUS) imaging probe was integrated into a commercial white light endoscope. AOUS imaging probes are ideally suited for this: they can be highly miniature (sub-millimetre diameter) [4], offer high bandwidths, resolution, and penetration (several centimetres) [5], and have previously been successfully demonstrated in vivo [6]. In addition, miniature fibre-optic imaging probes can be designed to be highly directional [7], which alleviates the strict positioning precision (better than $15 \mu \mathrm{m}$ ) required for accurate image reconstruction [8]. Through the imaging of a tissue-mimicking phantom, we demonstrate the first realtime, freehand, 3D and co-registered multimodal interventional imaging with an instrument combining white light endoscopy and AOUS imaging. 


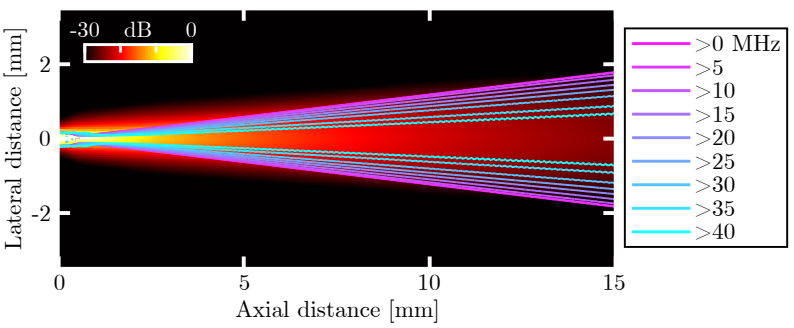

Fig. 1. Simulated pressure field emitted by a circular piston transducer with a diameter of $400 \mu \mathrm{m}$. The heat map shows the maximum amplitude pressure field emitted when the full bandwidth is used. The colour-coded contours indicate the full-width-at-half-maximum of the pressure distribution as a function of axial distance (and are representative of the lateral resolution) when a high-pass filter of varying cut-on frequency (listed in the legend) is applied. Pressure fields were modelled with the FOCUS toolbox [12].

\section{METHODS}

\section{A. All-optical ultrasound imaging}

All-optical ultrasound imaging probes utilise light to both generate and detect ultrasound. Ultrasound is generated via the photoacoustic effect: excitation light is delivered to an optically absorbing coating, resulting in an increase in temperature, subsequent thermoelastic expansion of the coating, and finally the generation of an ultrasound wave [9]. Backscattered ("pulse-echo") ultrasound waves are detected using optically resonant structures such as Fabry-Pérot cavities [10] or microring resonators [11], the reflectivity of which is modulated by impinging ultrasound waves.

In this work, highly directional ultrasound was generated at the tip of an optical fibre (core diameter: $400 \mu \mathrm{m}$; FT400EMT, Thorlabs, Germany) coated with a nano-composite of polydimethylsiloxane (PDMS; MED-1000, Polymer Systems Technology, UK) and functionalised reduced graphene oxide (805084, Sigma Aldrich, UK). A pulsed laser (wavelength: $1064 \mathrm{~nm}$, pulse energy: $40 \mu \mathrm{J}$, pulse duration: $1.6 \mathrm{~ns}$; DSS1064 Q, Crylas, Germany) was used to excite the ultrasound source. Back-scattered signals were detected by a fibre-optic detector (fibre type: SMF-28) comprising a planoconcave Fabry-Pérot cavity [10], which was interrogated by a wavelength-tunable CW laser (power: $20 \mathrm{~mW}$; TSL-550, Santec, UK) tuned to the sensor's bias point. The two fibre-optic transducers were assembled into an imaging probe (overall diameter: $800 \mu \mathrm{m}$ ) using heat shrink.

A-scans were continuously recorded at a rate of $100 \mathrm{~Hz}$, pre-amplified (20 dB; DHPVA-200, Femto, Germany), digitised (sample frequency: $125 \mathrm{MHz}$; M4i.4420-x8, Spectrum, Germany), and high-pass filtered to improve the directionality (and hence lateral resolution) of the ultrasound source (Fig. 1). A cut-on frequency of $15 \mathrm{MHz}$ was chosen as a compromise between directivity and signal-to-noise ratio of the recorded A-scan.

\section{B. Multimodal endoscopy}

The optical ultrasound imaging probe was inserted into the instrument channel of a commercially available urethroscope (17F; Hopkins 27035 BA 30 , Kalr Storz, Germany), which

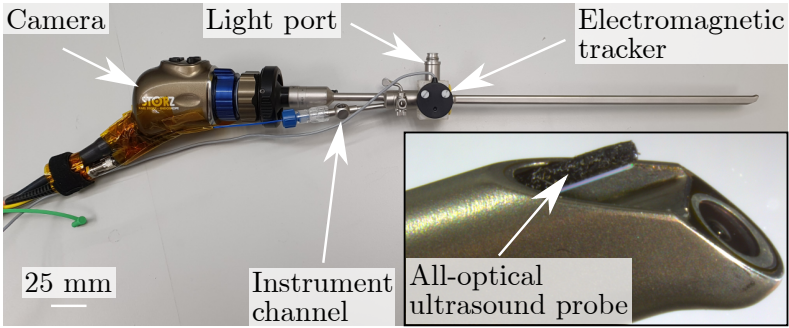

Fig. 2. Photograph of the endoscope used to perform multimodal alloptical ultrasound (AOUS) and white light imaging. A fibre-optic AOUS imaging probe was inserted into the instrument channel of a commercial urethroscope. The position and pose of the instrument tip were registered using an electromagnetic tracker, which was affixed to the urethroscope using a custom 3D-printed mount. Insert: distal end of the instrument, showing the white light endoscopy optics and the AOUS imaging probe housed within the instrument channel.

was equipped with a digital camera (IMAGE1 S H3-Z TH 100, Kalr Storz, Germany) and spatially calibrated [13]. White light (Cold Light Fountain Power LED 175 SCB, Kalr Storz, Germany) was delivered coaxially through a flexible light guide, and frames from the camera were grabbed in full HD (Magewell USB Capture SDI Gen 2, Nanjing Magewell Electronics, China) at a frame rate of $30 \mathrm{~Hz}$. To register the position and pose of the urethroscope tip, an electromagnetic (EM) tracker (update rate: $40 \mathrm{~Hz}$, RMS error: $0.80 \mathrm{~mm} /$ $0.7^{\circ}$; Aurora with Tabletop field generator, NDI, Canada) was attached to the endoscope shaft via a custom 3D-printed mount. The spatial offset between the uretrhoscope tip and EM tracker was determined via hand-eye calibration [14]. A photograph of the assembled urethroscope is shown in Fig. 2.

\section{Hardware communication}

Data acquisition, instrument synchronisation and image display were performed using a custom Robotics Operating System (ROS) [15] interface, which continuously acquired and time-stamped the endoscopy frames, ultrasound A-scans and tracker position at different rates. Endoscopy frames were continuously updated and displayed in real-time. The ultrasound A-scans were thresholded to eliminate noise, and spatially mapped along a line defined by the position and pose of the endoscope tip and an assumed speed of sound of $1500 \mathrm{~m} / \mathrm{s}$. Processed consecutive A-scans were added in real-time to a $3 \mathrm{D}$ point cloud, where a voxel filter was used to average the intensities and positions of points located within a $1 \mathrm{~mm}^{3}$ volume. This limited the number of points simultaneously displayed, and thus improved the frame rate (ca. $25-30 \mathrm{~Hz}$ ). The AOUS point cloud was displayed, in real-time, both separately and as a spatially-registered overlay on the endoscopy image. Endoscopy, tracking and AOUS data were continuously acquired, time-stamped, and recorded into a ROS bag [16] for additional off-line processing.

\section{Imaging experiment}

The addition of an EM tracker allowed for freehand operation of the multimodal endoscope. To demonstrate this capability, a tissue-mimicking phantom (measuring $12 \mathrm{~cm} \times 12 \mathrm{~cm}$; 
a)

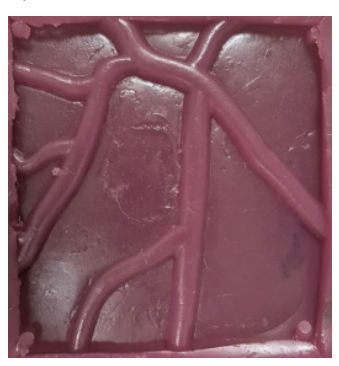

b)

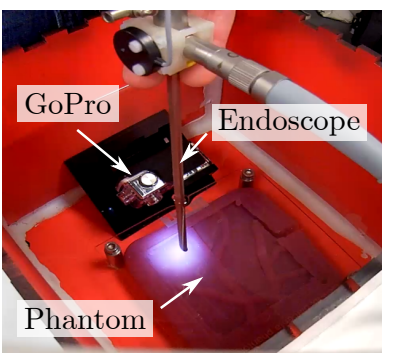

c)

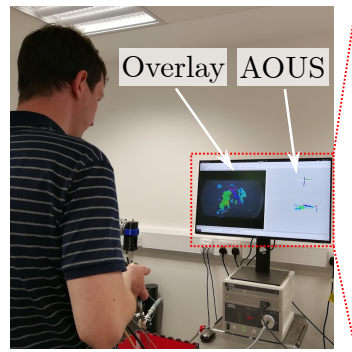

d)

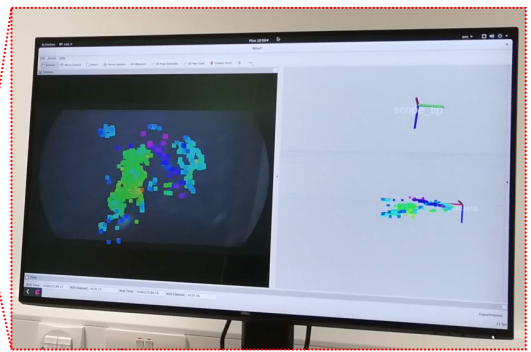

Fig. 3. Photographs of the imaging approach. a) The top surface of a tissue-mimicking phantom based on the vasculature on a human placenta. b) External view of the water bath used to perform the experiments. A GoPro camera was submerged to provide an underwater view during the experiment. c) The operator (EJA) was viewing a screen displaying a real-time, video-rate visualisation of both the 3D all-optical ultrasound (AOUS) point cloud (right half of screen) and a spatially registered overlay of the AOUS image over the white light endoscopy image (left half). The vertical (height) coordinate of the point cloud was colour-coded. d) Close-up of the operator screen.

Fig. 3(a)), modelled after the vasculature of a human placenta, was prepared using gelwax [17] and submerged in a water bath. The endoscope was then manually scanned across the surface of this phantom at a distance of approximately $1.5 \mathrm{~cm}$, while continuously recording white light images, AOUS data, and position and pose information. Real-time data acquisition, processing and display enabled direct assessment of the scanned image, which allowed the operator to identify areas of incomplete coverage and to navigate back to these areas to locally improve the image quality. Two video recordings were made during the experiment: a GoPro Hero (GoPro, Ca, USA) was submerged to provide an underwater view, and a separate camera (Action CT6500, Crosstour, China) was placed externally to provide a time-lapse recording of the manual scanning. The phantom, its mounting within the water bath, and the imaging paradigm are shown in Fig. 3.

\section{RESULTS}

Data recorded during the experiment were post-processed off-line in MATLAB (2019b; The MathWorks, MA, USA) using the same signal processing steps as those performed in real-time. Videos of the white-light endoscope image, 3D AOUS image and submerged camera were synchronised and combined off-line, and demonstrated how a high-quality 3D AOUS image could be progressively built up in a matter of minutes ( $c a .5$ minutes for this experiment). During the experiment the endoscope was repeatedly retracted from the phantom (at time points $3 \mathrm{~m} 0 \mathrm{~s}, 3 \mathrm{~m} 35 \mathrm{~s}, 4 \mathrm{~m} 0 \mathrm{~s}$ and $4 \mathrm{~m} 24 \mathrm{~s}$ ) to allow the operator to identify areas of poor coverage, reposition the instrument, and continue piece-wise scanning without the need to recalibrate the instrument. The full postprocessed video of this experiment can be found on-line (youtu.be/AniNd5AACZs); snapshots at different time points are shown in Fig. 4.

\section{Discussion AND CONCLUSION}

In this work, we present the first co-registered, freehand and multimodal images combining all-optical ultrasound (AOUS) with white-light endoscopy. This was achieved by inserting an AOUS imaging probe into the instrument channel of a commercial urethroscope, which required no changes to the form factor or function of the urethroscope. Due to its small dimensions, the AOUS imaging probe left several millimetres of the instrument channel free for other therapeutic or diagnostic instruments. The addition of an EM tracker enabled freehand operation, accurate registration between the imaging modalities, and piece-wise acquisition of the AOUS image. No adverse effects on the performance of the endoscope were observed.

AOUS imaging was performed using a highly directional source, which confined sensitivity to just the line along the long axis of the instrument. This approach alleviated the strict positioning accuracy requirements associated with common image reconstruction algorithms [8], and allowed for direct spatial mapping of the A-scan. High-pass filtering was applied to improve the source directivity; however, stricter filtering could be applied to further enhance the directivity, and hence lateral resolution, at the expense of a reduction in A-scan signal-to-noise ratio and penetration.

The use of a highly directional source introduces two main issues. First, acoustical scattering and refraction in inhomogeneous media could give rise to spatial distortions and acoustic shadowing, the latter of which accounts for the gaps observed around the blood vessels in the AOUS image in Fig. 4. Second, specular reflectors (such as near-straight watertissue boundaries) can under large angles of incidence reflect ultrasound away from the optical ultrasound receiver, and thus require the endoscope to be held approximately normal to the tissue surface. This restriction can be alleviated in future work by incorporating miniature actuator components, such as McKibben muscles [18], that can control the orientation of the AOUS A-scan relative to the endoscope.

In future work, the feasibility of real-time mosacking [19] will be explored to alleviate the limited aperture (ca. $3 \mathrm{~mm}$ ) offered by the endoscope, which will greatly add to the value of the endoscopy-AOUS overlay image. In addition, a tissuemimicking phantom with sub-surface features will be considered to assess the penetration depth and visualise features not visible using white-light endoscopy. Finally, the remaining space available in the urethroscope instrument channel will be 

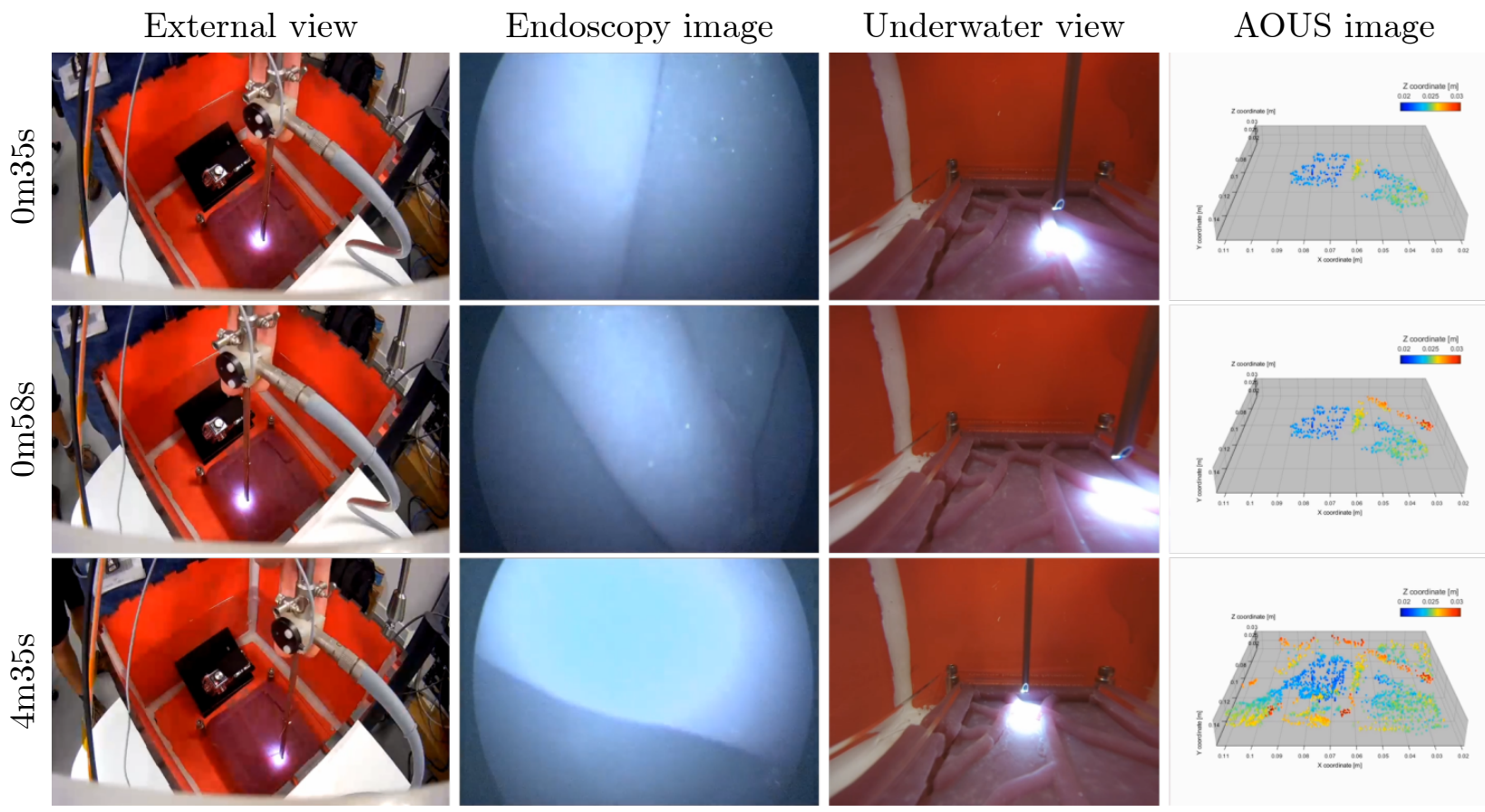

Fig. 4. Snapshots of the combined video obtained after off-line processing of data acquired during freehand and multimodal imaging. The panels show, from left to right, the view recorded using an externally positioned camera; the white-light endoscopy video feed; the underwater view from the submerged camera; and the progressive build-up of the 3D all-optical ultrasound (AOUS) image, where the vertical (height) axis was colour-coded. These video frames were obtained at (from top to bottom) $0 \mathrm{~m} 35 \mathrm{~s} ; 0 \mathrm{~m} 58 \mathrm{~s}$; and $4 \mathrm{~m} 35 \mathrm{~s}$ from the start of the experiment. The full video of this experiment can be found on-line (youtu.be/AniNd5AACZs).

used to add therapeutic capabilities, such as photo- or radiofrequency ablation.

To the authors' knowledge, this work presents the first demonstration of multimodal (AOUS combined with white light), real-time and co-registered 3D image acquisition, processing and display. Housed within a commercially available endoscope, the presented imaging probe and system show great promise to significantly improving image guidance during interventional surgery.

\section{REFERENCES}

[1] Amanda Cole, Phill O'Neill, Chris Sampson, and Paula Lorgelly. Barriers to uptake of minimal access surgery in the united kingdom. $O H E$ Consulting Report, London: Office of Health Economics, 2018.

[2] Xue Li, Xue-Ning Zhang, Xiao-Dong Li, and Jin Chang. Multimodality imaging in nanomedicine and nanotheranostics. Cancer biology \& medicine, 13(3):339, 2016.

[3] Francisco PM Oliveira and Joao Manuel RS Tavares. Medical image registration: a review. Computer methods in biomechanics and biomedical engineering, 17(2):73-93, 2014.

[4] Richard J Colchester, Edward Z Zhang, Charles A Mosse, Paul C Beard, Ioannis Papakonstantinou, and Adrien E Desjardins. Broadband miniature optical ultrasound probe for high resolution vascular tissue imaging. Biomedical Optics Express, 6(4):1502-1511, 2015.

[5] Sacha Noimark, Richard J Colchester, Radhika K Poduval, et al Polydimethylsiloxane composites for optical ultrasound generation and multimodality imaging. Advanced Functional Materials, 28(9), 2018.

[6] Malcolm C Finlay, Charles A Mosse, Richard J Colchester, et al. Through-needle all-optical ultrasound imaging in vivo: a preclinical swine study. Light: Science \& Applications, 6(12):e17103, 2017.

[7] Richard J Colchester, Erwin J Alles, and Adrien E Desjardins. A directional fibre optic ultrasound transmitter based on a reduced graphene oxide and polydimethylsiloxane composite. Applied Physics Letters, 114(11):113505, 2019.
[8] Erwin J Alles, Sacha Noimark, Edward Zhang, Paul C Beard, and Adrien E Desjardins. Pencil beam all-optical ultrasound imaging. Biomedical Optics Express, 7(9):3696-3704, 2016.

[9] Paul C Beard. Biomedical photoacoustic imaging. Interface Focus, 1(4):602-631, 2011

[10] James A Guggenheim, Jing Li, Thomas J Allen, et al. Ultrasensitive plano-concave optical microresonators for ultrasound sensing. Nature Photonics, 11(11):714-719, 2017.

[11] Suzanne M Leinders, Wouter J Westerveld, J Pozo, et al. A sensitive optical micro-machined ultrasound sensor (OMUS) based on a silicon photonic ring resonator on an acoustical membrane. Scientific Reports, $5,2015$.

[12] Robert J. McGough. FOCUS: Fast Object-Oriented C++ Ultrasound Simulator, www.egr.msu.edu/ fultras-web/index.php. Accessed: 24 May 2020.

[13] Zhengyou Zhang. A flexible new technique for camera calibration. IEEE Transactions on pattern analysis and machine intelligence, 22(11):1330$1334,2000$.

[14] Roger Y Tsai and Reimar K Lenz. A new technique for fully autonomous and efficient $3 \mathrm{D}$ robotics hand/eye calibration. IEEE Transactions on robotics and automation, 5(3):345-358, 1989.

[15] Morgan Quigley, Ken Conley, Brian Gerkey, et al. ROS: an open-source robot operating system. In ICRA workshop on open source software, volume 3, page 5. Kobe, Japan.

[16] ROS: Robot Operating System. www.ros.org. Accessed: 28 July 2020

[17] Efthymios Maneas, Wenfeng Xia, Daniil I Nikitichev, et al. Anatomically realistic ultrasound phantoms using gel wax with 3D printed moulds. Physics in Medicine \& Biology, 63(1), 2018.

[18] Alain Devreker, Benoit Rosa, Adrien Desjardins, et al. Fluidic actuation for intra-operative in situ imaging. In Proceedings IEEE/RSJ IROS, pages 1415-1421. IEEE, 2015.

[19] Sophia Bano, Francisco Vasconcelos, Marcel Tella Amo, et al. Deep sequential mosaicking of fetoscopic videos. In International Conference on Medical Image Computing and Computer-Assisted Intervention, pages 311-319. Springer. 